\title{
Diagnostisches und didaktisches Handeln verbinden: Entwicklung eines Prozessmodells auf der Grundlage von Erkenntnissen aus der pädagogischen Diagnostik und der Förderdiagnostik
}

\author{
Elisabeth Moser Opitz $(\mathbb{D}$
}

Eingegangen: 22. Dezember 2020 / Angenommen: 25. Februar 2022 / Online publiziert: 8. März 2022

(C) Der/die Autor(en) 2022

Zusammenfassung Für Lehrkräfte ist die Anpassung der unterrichtlichen Aktivitäten an die Lernvoraussetzungen der Schülerinnen und Schüler und damit verbunden das diagnostische und didaktische Handeln zentral. In der Mathematikdidaktik finden sich zu dieser Aufgabe vereinfacht dargestellt zwei Zugänge: Zum einen orientiert sie sich an Konzepten und Modellen aus der pädagogischen Diagnostik bzw. der empirischen Unterrichtsforschung, zum anderen an dem aus der Sonderpädagogik stammendem Konzept der Förderdiagnostik. Die beiden Zugänge unterscheiden sich deutlich in den Zielsetzungen sowie in den Aufgabenfeldern. Diese Unterschiede wurden bisher nicht systematisch aufgearbeitet. Zudem wurde die Verbindung zwischen diagnostischem und didaktischem Handeln in beiden Zugängen wenig reflektiert, insbesondere hinsichtlich des dafür notwendigen Professionswissens der Lehrkräfte. Im Artikel werden ausgewählte Konzepte aus der pädagogischen Diagnostik sowie die Förderdiagnostik mit Blick auf das mathematische Lernen dargestellt und vergleichend diskutiert. Dabei zeigt sich, dass die Frage nach den Kriterien für die Urteilsbildung und für die didaktische Entscheidung sowohl in der Förderdiagnostik als auch der pädagogischen Diagnostik vernachlässigt wird. In einem Modell wird herausgearbeitet, dass in verschiedenen Phasen des Diagnose- und Förderprozesses (diagnostisches Handeln, Urteilsbildung, didaktische Entscheidung, didaktisches Handeln) jeweils spezifisch diagnostisches, fachliches und fachdidaktisches Professionswissen notwendig ist, um theoriegeleitet vorgehen zu können. Der Artikel leistet einen Beitrag zur Konzeptualisierung von diagnostischem und didaktischem Handeln bezogen auf das mathematische Lernen.

Elisabeth Moser Opitz $(\square)$

Institut für Erziehungswissenschaft, Universität Zürich, Zürich, Schweiz

E-Mail: elisabeth.moseropitz@uzh.ch 
Schlüsselwörter Pädagogische Diagnostik · Förderdiagnostik · Diagnostisches Handeln · Didaktisches Handeln · Urteilsbildung · Professionelles Wissen von Lehrkräften

MESC Codes $\mathrm{B} 10 \cdot \mathrm{B} 50 \cdot \mathrm{C} 10 \cdot \mathrm{C} 90 \cdot \mathrm{D} 20 \cdot \mathrm{D} 70 \cdot \mathrm{Q} 70$

\title{
The Interrelationship of Diagnosis and Teaching: Developing a Process Model Incorporating Knowledge of Educational Diagnostics and Individual Education Plans
}

\begin{abstract}
One of the most important skills teachers must have is the ability to adapt classroom teaching to suit pupil abilities, which involves both diagnosis and teaching. Researchers in the field of mathematics education tend to take one of two theoretical approaches. One is based on pedagogical diagnostics and research into teaching quality. The other has its origin in special education and focuses on individual education plans, "Förderdiagnostik" in German. These approaches have divergent priorities and objectives and their differences have yet to be systematically analyzed. The interrelationship between diagnosis and teaching and the professional knowledge these activities require has also not been considered in detail. This paper reviews selected aspects of the pedagogical diagnostic approach and the "Förderdiagnostik" approach as they apply to mathematics education. The analysis reveals that there is often no theoretical basis for evaluating educational assessments and teacher decision making. A process model is developed demonstrating that in each phase-diagnosis, educational assessment, educational decision and teaching - the relevant professional knowledge (diagnostic knowledge, mathematical knowledge for teaching) is important. In addition, the results indicate that the skills required to fulfill one objective, such as the assessment of pupil achievement levels, can be very different from those required for assessing a pupil's individual support requirements. This paper makes an important contribution to the conceptual framework of the relationship between diagnosis and mathematics teaching.
\end{abstract}

Keywords Educational diagnostics - Individual education plans · Diagnosis · Teaching · Educational assessment $\cdot$ Teacher professional knowledge

\section{Einleitung}

Individuelle Förderung ist gemäß Klieme und Warwas (2011, S. 805) zu einem ,zentralen Topos des öffentlichen Bildungsdiskurses in Deutschland“ geworden und die Anpassung der unterrichtlichen Aktivitäten an die Lernvoraussetzungen der Schülerinnen und Schüler gilt als eine wichtige Aufgabe von Lehrkräften (Brühwiler 2014). Ein solch adaptives Handeln wurde von Ingenkamp (1985, S. 18) im Rahmen der pädagogischen Diagnostik als ,die enge Verzahnung von diagnostischen Schritten und darauf aufbauenden didaktischen Eingriffen“ beschrieben. Der Sonderpädagoge Kobi (1977) hat für dieses Vorgehen die sogenannte „Förderdiagnostik“ konzeptualisiert; eine Diagnostik, die nicht die Selektion und die Zuweisung zu einer be- 
stimmten Schulform zum Ziel hat, sondern durch die Verbindung von Diagnose und pädagogischem bzw. didaktischem Handeln ${ }^{1}$ die individuelle Förderung anstrebt. In der Mathematikdidaktik wird auf beide Zugänge - auf die pädagogische Diagnostik und die Förderdiagnostik - Bezug genommen. Zum einen orientieren sich die Forschenden an den Modellen aus der pädagogischen Diagnostik (Herppich et al. 2017) oder der Psychologie des Urteilens (Loibl et al. 2020). Zudem wurden von Forschenden aus der pädagogischen Psychologie und der Mathematikdidaktik viele Untersuchungen zur Diagnosekompetenz von Mathematiklehrkräften durchgeführt (z.B. Anders et al. 2010; Heinrichs und Kaiser 2018; Hoffmann und Böhme 2014; Hoth et al. 2016; Philipp 2018; Karst et al. 2014). Zum anderen ist ein enger Bezug zur Förderdiagnostik zu erkennen. Von Mathematikdidaktikerinnen und -didaktikern wurden Instrumente für die Erfassung des Lernstandes und für die Planung der Individualdiagnostik und der anschließenden Förderung entwickelt und erprobt (z.B. Prediger et al. 2017; Hußmann und Prediger 2014a, b; Kaufmann und Wessolowski 2006; Fricke und Streit-Lehmann 2015; Peter-Koop 2015; Scherer 2018a, b, 2019).

Die Auseinandersetzung mit der Thematik der Diagnose und Förderung erfolgt somit in der deutschsprachigen Mathematikdidaktik - vereinfacht gesagt - mit zwei unterschiedlichen Zugängen. Diese stehen jedoch getrennt nebeneinander, die jeweiligen Diskurse finden isoliert voneinander statt und eine systematische Auseinandersetzung damit fehlt bisher. Diese ist jedoch aus verschiedenen Gründen wichtig. Erstens ist es grundsätzlich von wissenschaftlichem Interesse, solche parallelen Diskurse zu reflektieren und Unterschiede und Gemeinsamkeiten herauszuarbeiten. Reflexionen zur pädagogischen Diagnostik und zur Förderdiagnostik sind insbesondere auch deshalb interessant, weil zentrale Publikationen zur pädagogischen Diagnostik (Ingenkamp 1985; Klauer 1978; Schrader 1989) und die ersten Publikationen zur Förderdiagnostik (Bundschuh 1985; Kobi 1977) zeitgleich erschienen sind. Allerdings haben diese Autoren - mit Ausnahme von Ingenkamp (1985) nicht aufeinander Bezug genommen. Zweitens ist eine Diskussion der beiden Zugänge für die Mathematikdidaktik relevant hinsichtlich der Verbindung von diagnostischem und didaktischem Handeln. Diagnostisches Handeln wird im Folgenden in Anlehnung an Ingenkamp (1985) vereinfacht als der Prozess der Diagnosestellung bezeichnet, das didaktische Handeln als die darauf abgestimmten unterrichtlichen Aktivitäten Auch hier sind bei beiden Zugängen Forschungslücken festzustellen. In den mathematischen Lernstandserfassungen und Diagnoseinstrumenten, die sich an der Förderdiagnostik orientieren, ist die Verbindung zwischen diagnostischem und didaktischem Handeln praktisch bedeutsam und wird hergestellt, indem sowohl Diagnoseaufgaben, Förderhinweise und entsprechende Aufgaben zur Verfügung gestellt werden. Dies erfolgt jedoch auf einer konkreten und praxisbezogenen Ebene. Mit grundlegenden Fragen zur Förderdiagnostik bzw. zu Fragen zur Verbindung von diagnostischem und didaktischem Handeln befassen sich nur wenige Beiträge. In diesen (z. B. Hößle et al. 2017; Scherer und Moser Opitz 2010; Wember 2018) wird in Anlehnung an Schlee (1985a, b, 2004, 2008) die Ableitung von Förderzielen aus einer Diagnose kritisiert und damit verbunden die Frage gestellt, auf welcher

\footnotetext{
${ }^{1}$ Da der Fokus auf der Mathematikdidaktik liegt, wird im Folgenden der Begriff „,didaktisches Handeln“ als Synonym für pädagogisches und didaktisches Handeln verwendet.
} 
Grundlage diagnostische und didaktische Entscheidungen gefällt werden können. Es bleibt in den erwähnten Publikationen jedoch bei der Problematisierung dieser Thematik und insbesondere fehlt die Auseinandersetzung mit der Frage, welche Rolle dem Professionswissen der Lehrkräfte in den einzelnen Phasen des Diagnose- und Förderprozesses zukommt.

In den Publikationen aus der Mathematikdidaktik, die sich an der pädagogischen Diagnostik und den entsprechenden Modellen orientieren, spielen Überlegungen zur Verbindung des diagnostischen und didaktischen Handelns der Lehrkräfte oft eine untergeordnete Rolle. Der Schwerpunkt liegt häufig auf dem diagnostischen Handeln, insbesondere auf dem Prozess der Urteilsbildung (z. B. Ingenkamp 1985; Gerich et al. 2017; Herppich et al. 2017, 2018; Rieu et al. 2022; Loibl et al. 2020). Für diesen werden spezifische diagnostische Kompetenzen ${ }^{2}$ der Lehrkräfte als wichtig erachtet. Vernachlässigt wird häufig die Frage, auf welcher Grundlage Entscheidungen getroffen werden und wie aus diagnostischen Informationen pädagogische Zielsetzungen abgeleitet werden können. Wenn es jedoch darum geht, das diagnostische und didaktische Handeln der Lehrkräfte zu verbessern (Helmke 2015; Kultusministerkonferenz 2014), ist es wichtig, sich mit dieser Frage und der Verbindung von diagnostischem und didaktischem Handeln auseinanderzusetzen.

Dieser Beitrag setzt sich deshalb das Ziel, die Verbindung - oder wie Ingenkamp (1985) schreibt, die Verzahnung - von diagnostischem und didaktischem Handeln in der Förderdiagnostik und der pädagogischen Diagnostik im Kontext der Mathematikdidaktik zu analysieren und zu reflektieren. Dabei interessiert erstens, auf welcher Grundlage diagnostische Informationen bewertet und didaktische Entscheidungen getroffen werden können. Zweitens erfolgt die Auseinandersetzung mit der Frage, welches Professionswissen Lehrkräfte für diese Aufgabe benötigen. Diese Überlegungen münden in ein Prozessmodell, in dem die Verbindung von diagnostischem und didaktischem Handeln unter Berücksichtigung des in den einzelnen Phasen des Diagnoseprozesses erforderlichen professionellen Wissens dargestellt wird.

Im Folgenden werden zuerst die Zielsetzungen und Merkmale der Förderdiagnostik sowie der pädagogischen Diagnostik mit Blick auf die Verbindung von diagnostischem und didaktischem Handeln im mathematikdidaktischen Kontext dargestellt.

\section{Förderdiagnostik und pädagogische Diagnostik: Beschreibung der Konzepte und Zielsetzungen}

\subsection{Förderdiagnostik}

\subsubsection{Zielsetzungen und Merkmale}

Das Konzept der Förderdiagnostik besteht seit dem Ende der 1970er-Jahre und hat seinen Ausgangspunkt in der Sonderpädagogik. Kobi (1977) kritisierte damals mit

\footnotetext{
2 Die Begriffe diagnostische und didaktische Kompetenzen und diagnostisches und (fach-)didaktisches Wissen werden in den verschiedenen zitierten Studien nicht einheitlich verwendet. Bevor in Kapitel 4 eine eigene Begriffsklärung erfolgt, werden jeweils die Begriffe aus den einzelnen Studien übernommen.
} 
28 Thesen die psychologische „Einweisungsdiagnostik“ heftig und stellte 28 plakative Thesen auf, die er unter dem Begriff der „Förderdiagnostik“ zusammenfasste. Das Paradigma der Förderdiagnostik sei die Interaktion zwischen Lehrenden und Lernenden, sie finde ihre Zweckbestimmung in der Förderung und nicht in der Selektion; sie sei an intra-individuellen Unterschieden und in erster Linie am Subjekt und den Lernenden interessiert; sei prozesshaft; Förderung und pädagogische Intervention würden eine untrennbare Einheit bilden, usw. Diese Kritik an der psychologischen Diagnostik wurde in der Sonderpädagogik - und später in der inklusiven Pädagogik - bereitwillig aufgenommen (Breitenbach 2020) und führte zu Konzepten und Prozessmodellen, die diagnostisches und didaktisches Handeln durch die Erstellung von Förderplänen miteinander verbinden (Buholzer 2014; Luder und Kunz 2014; Popp et al. 2017).

Kennzeichnend für diese Modelle ist erstens, dass die Zielsetzung des förderdiagnostischen Vorgehens immer die Förderung von einzelnen Individuen in verschiedenen Entwicklungsbereichen (sozial, kognitiv, motorisch, fachlich usw.) ist. Die Beurteilung im Sinn von Notengebung, die Bewertung von leistungsrelevanten Merkmalen gemessen an der Sozialnorm mit dem Ziel zur Zuweisung zu bestimmten Schulformen und das Erstellen einer Rangreihenfolge der Schülerinnen und Schüler nach Leistung wird ausgeschlossen (Bundschuh 1985; Eggert und Reichenbach 2007). Der Fokus liegt zudem auf der individuellen Förderung und der Unterrichtskontext wird kaum berücksichtigt. Zweitens wurden die Testdiagnostik, die damit verbundene Standardisierung sowie die Anwendung der klassischen Gütekriterien zum Teil heftig kritisiert und als unnötig erachtet (z.B. Eggert und Reichenbach 2007). Gefordert werden ,qualitative Gütekriterien“ (ebd., S. 77) wie die intersubjektive Übereinstimmung von mehreren Beobachtenden bzw. die Konsensfindung im Team, die wertfreie Beschreibung des Beobachteten, usw. Drittens wird das förderdiagnostische Vorgehen häufig mit Prozess- bzw. Kreismodellen mit den Phasen Diagnose, Förderplanung, Förderung und Evaluation modelliert (z. B. Buholzer 2014; Bundschuh 2007; Luder und Kunz 2014; Popp et al. 2017). In Abb. 1 wird ein solches Modell exemplarisch dargestellt.

Die Implementation der Förderdiagnostik erfolgt in der Regel mit der Erstellung von Förderplänen (Popp et al. 2017). Empirische Untersuchungen zeigen jedoch, dass sich dies als sehr anspruchsvoll gestaltet und dass die Umsetzung oft wenig erfolgreich ist. Müller et al. (2017) referieren in ihrer Literaturrecherche den Nutzen von Förderplänen aus der Sicht von Lehrkräften. Die Ergebnisse zeigen, dass die negativen Einschätzungen von Förderplänen eher überwiegen. Kritisiert wird beispielsweise, dass die Arbeit mit Förderplänen zeitintensiv und für den Alltag wenig tauglich sei. Paccaud und Luder (2017) kommen auf der Grundlage einer Analyse von Förderplänen zum Schluss, dass die Qualität der dort formulierten Lernziele niedrig sei. Moser Opitz et al. (2019) untersuchten den Einsatz von Förderplänen in inklusiven und separativen Klassen auf der Primar- und Sekundarstufe. Die Ergebnisse zeigen, dass die Erstellung von Förderplänen von den Befragten häufig als eine bürokratische Notwendigkeit gesehen wird, und die Pläne vor allem eine Dokumentations- und Legitimationsfunktion haben, beispielsweise wenn es um Schullaufbahnentscheidungen geht. Der eigentlich beabsichtigte Aspekt der Förderung scheint oft im Hintergrund zu stehen. 
Abb. 1 Prozessmodell der Förderplanung. (Eigene Darstellung nach Luder und Kunz 2014, S. 58)

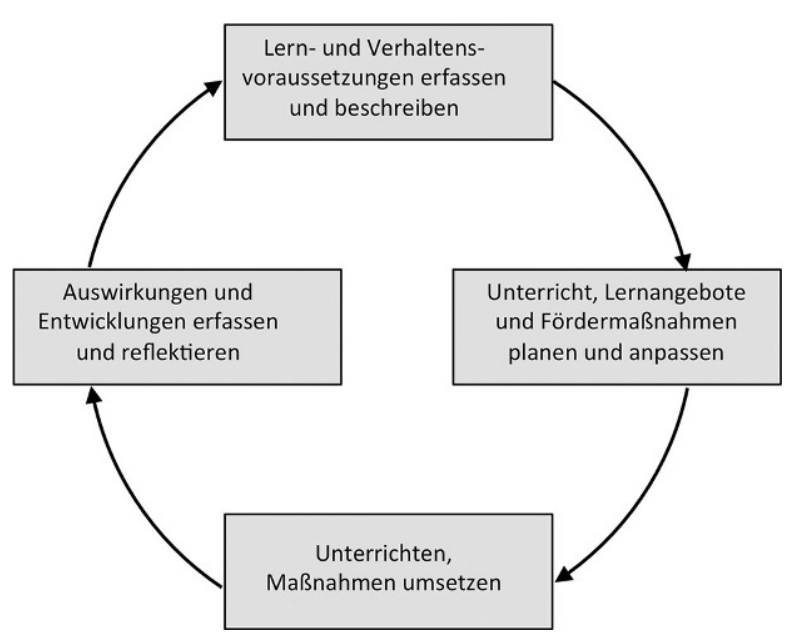

\subsubsection{Diagnostisches und didaktisches Handeln in der Förderdiagnostik}

Zentrales Anliegen der Förderdiagnostik ist, wie im Modell in Abb. 1 dargestellt, die enge Verbindung von diagnostischem und didaktischem Handeln. Ausgehend von beobachteten Lern- und Verhaltensweisen der Schülerinnen und Schüler sollen Lern- und Förderangebote geplant werden. Genau dieser Punkt wurde jedoch aus erkenntnistheoretischer Sicht insbesondere von Schlee (1985a, b, 2004, 2008) kritisiert. Hauptkritikpunkt ist nach Schlee, dass das Konzept der Förderdiagnostik einen naturalistischen Fehlschluss bzw. einen Sein-Sollen-Fehlschluss (Schlee 2002) enthalte, wenn aufgrund von Beobachtungen Fördermaßnahmen festgelegt würden. Deskription im Sinn der Beschreibung der Lernausgangslage und Präskription - das Festlegen von Zielsetzungen - würden vermischt. Schlee (ebd.) erläutert diese mit folgendem Beispiel:

Die Aufstellung eines Förderplans ergibt sich weder als Anregung noch als Verpflichtung aus den diagnostischen Resultaten, sondern stellt wiederum einen eigenständigen Konstruktionsprozess dar. ... Nicht die diagnostischen Daten führen zu Fördervorschlägen, sondern das bereits vor der Diagnostik vorhandene Handlungs- und Planungswissen der Personen. (Schlee 2002, S. 186; Hervorhebung im Original)

Schlee (1985a) führt aus, dass sich Vorstellungen über Veränderungs- oder Förderziele - die Präskription - erst ergeben, wenn eine spezifische Situation bewertet worden ist und plädiert deshalb dafür, dass die Didaktik der Diagnostik zeitlich und logisch über- bzw. vorgeordnet sei: Die Didaktik liefere Bezugspunkte für die Diagnostik, diese wiederum kontrolliere und evaluiere die Didaktik (Schlee 1985b, 2002). Für die Festlegung von Fördermaßnahmen sind deshalb nach Schlee (2007, S. 178) „klar formulierte theoretische Positionen“ zentral. Den Überlegungen von Schlee folgend umschreibt Wember (1998) Diagnosen mit vier Merkmalen: Sie beschreiben momentane Zustände in selektiver Weise, sie sind grundsätzlich immer 
mit Wertentscheidungen verbunden, sie sind theoriebestimmt - durch wissenschaftliche Theorien oder Alltagstheorien - und sie sind „deskriptive Sätze, die allein und für sich genommen keine anzustrebenden Ziele begründen und die allein und für sich genommen auch keine zielführenden Handlungen legitimieren, fundieren und optimieren können“ (ebd., S. 110).

\subsubsection{Förderdiagnostik im Kontext der Mathematikdidaktik}

Mathematikdidaktische Publikationen zum Thema Förderdiagnostik beziehen sich häufig implizit, manchmal auch explizit auf Literatur zum Thema Förderdiagnostik und/oder Förderpläne aus der Sonderpädagogik (z. B. Peter-Koop 2015; Fricke und Streit-Lehmann 2015). In den Handreichungen für die Praxis (z. B. Prediger et al. 2017; Hußmann und Prediger 2014a, b; Scherer 2018a, b, 2019) ist die Verbindung zwischen diagnostischem und didaktischem Handeln sichtbar, indem Diagnoseaufgaben, dazu passende Förderhinweise sowie konkrete Aufgabenstellungen zur Verfügung gestellt werden. Dieser Zusammenhang zwischen diagnostischem und didaktischem Handeln wird häufig indirekt thematisiert, indem die den konkreten Hinweisen zugrunde liegenden didaktischen Prinzipien bzw. der fachdidaktische Hintergrund (kurz) dargestellt werden (z.B. Prediger et al. 2017; Hußmann und Prediger 2014a, b; Kaufmann und Wessolowski 2006; Scherer 2018a, b, 2019).

Die Problematik der Ableitung von Förderzielen aus der Diagnose bzw. aus Beobachtungen wird auch in mathematikdidaktischen Publikationen (Hößle et al. 2017; Moser Opitz und Nührenbörger 2015; Scherer und Moser Opitz 2010; Wember 2018) diskutiert. Moser Opitz und Nührenbörger (2015) fordern beispielsweise, dass einer Diagnose fachliche und fachdidaktische Überlegungen vorausgehen müssen, die zum einen die Grundlage für die diagnostischen Fragen, die Entwicklung der Diagnoseaufgaben und die diagnostischen Analysen darstellen und zum anderen auch handlungsleitend sind für die Planung der Förderung. Hößle et al. (2017) beziehen sich auf Schlee (2008) und Wember (1998) und stellen fest, dass ,... eine Diagnostik nur dann hilfreich sein kann, wenn sie mit Blick auf fachdidaktische Konzepte und Theorien für die Entwicklung des Lernens der Schülerin bzw. des Schülers entwickelt wird" (Hößle et al. 2017, S. 20). Wember (2018, S. 239) spricht von ,diagnostisch-präskriptivem Unterricht“, der sich dadurch auszeichnet, dass ein „ein didaktisch zureichend begründeter Katalog von Schlüsselqualifikationen aufgestellt" werden soll, ,um anschließend prüfen zu können, welche Qualifikationen die einzelnen Schülerinnen und Schüler bereits erworben haben und welche sie erst erlernen müssen“ (ebd., S. 240).

Zusammenfassend kann festgestellt werden, dass schon der Begriff Förderdiagnostik deutlich macht, dass diagnostisches und didaktisches Handeln in engem Zusammenhang stehen. Damit verbunden ist jedoch die fehlende Unterscheidung von Deskription und Präskription und die Problematik des logischen Fehlschlusses bezüglich der Urteilsbildung und der didaktischen Entscheidung. Obwohl in mathematikdidaktischen Publikationen auf diese Problematik hingewiesen und gefordert wird, dass diagnostische Informationen ,theoriebasiert“ erhoben und interpretiert werden müssen, fehlen bisher systematische Überlegungen, welche „Theorien“ in welcher Phase des Prozesses von Diagnose und Förderung wichtig sind. 


\subsection{Pädagogische Diagnostik}

\subsubsection{Zielsetzungen und Merkmale}

Der pädagogischen Diagnostik werden verschiedene Aufgaben auf unterschiedlichen Ebenen zugewiesen. Nach Ingenkamp (1985) hat die pädagogische Diagnostik einerseits die Optimierung der individuellen Lernprozesse zum Ziel, andererseits auch die Feststellung von Lernergebnissen mit Blick auf den Übergang in verschiedene Schultypen oder Bildungswege (vgl. auch Klauer 1978; van Ophuysen 2010). Herppich et al. (2017) formulieren die Zielsetzung der pädagogischen Diagnostik allgemein als Informationsbasis für das Treffen von pädagogischen Entscheidungen. Dies kann einerseits auf Klassenebene erfolgen, wenn es z. B. darum geht, die Leistungen der Schülerinnen und Schüler möglichst genau einzuschätzen (Helmke 2015). Nach Schrader (2013) gehören dazu Notengebung, Versetzung, Übergangsempfehlungen, Unterrichtsplanung und -gestaltung sowie Schul- und Unterrichtsentwicklung. Es geht andererseits aber auch darum, die individuellen Lernvoraussetzungen von Lernenden zu erfassen, um anschließend individualisierte und gezielte Unterstützung anbieten zu können (Hoth et al. 2016). Das heißt, dass in der pädagogischen Diagnostik je nach Zielsetzung eine Ausrichtung an der Individualnorm, der Sozialnorm oder einer kriteriumsorientierten Norm (Lehrplan, Bildungsstandards) erfolgen kann. Kennzeichnend für die pädagogische Diagnostik ist zudem, dass zum Erreichen der genannten Zielsetzungen die diagnostischen Kompetenzen der Lehrkräfte als zentral erachtet werden. Entsprechend befassen sich der wissenschaftliche Diskurs und die entsprechenden Untersuchungen in der pädagogischen Diagnostik vorwiegend mit dieser Thematik.

Als wichtig erachtet und vielfach untersucht wurde im Anschluss an Helmke und Schrader (1987) die Akkuratheit einer Diagnose bzw. die Urteilsgenauigkeit. Helmke (2015) operationalisiert diese - in Anlehnung an Cronbach (Spinath 2005) - mit der Niveau-, der Streuungs- und der Rangordnungskomponente. Bei der Niveaukomponente geht es darum, ob eine Lehrkraft die durchschnittliche Leistung ihrer Klasse einschätzen kann. Die Streuungskomponente beinhaltet die Frage, ob die Einschätzung der Schwierigkeit von bestimmten Aufgaben den tatsächlichen Lösungen der Lernenden entspricht. Bei der Rangordnungskomponente geht es darum, Fähigkeitsabstufungen zwischen den Lernenden zu erkennen. Nach Helmke (2015, S. 134) ist dies das „Kernstück“ der diagnostischen Kompetenz. Bei der Akkuratheit liegt der Schwerpunkt somit auf der Einschätzung der einzelnen Schülerinnen und Schüler im Vergleich zu den Peers. Die dafür notwendige diagnostische Expertise von Lehrkräften umfasst dementsprechend die Verfügbarkeit von Methoden zur Einschätzung der Leistung von Lernenden, die Kenntnis von Urteilstendenzen und -fehlern usw. Gemäß Schrader (2013) ist eine fundierte methodische Absicherung der Urteile und damit verbunden ein hoher Anspruch an die Gütekriterien Validität, Objektivität und Reliabilität sehr wichtig, da pädagogische Diagnosen längerfristig als Grundlage für Übertrittsentscheidungen genutzt werden und sich somit auf die schulische Biografie einzelner Individuen auswirken. Ausgehend davon wird entsprechenden diagnostischen Kompetenzen der Lehrkräfte hohe Bedeutung zugeschrieben (ebd.; Herppich et al. 2017). 


\subsubsection{Diagnostisches und didaktisches Handeln in der pädagogischen Diagnostik}

Der Zusammenhang von diagnostischem und didaktischem Handeln wird in der pädagogischen Diagnostik von verschiedenen Autorinnen und Autoren unterschiedlich konzeptualisiert. Dies wird im Folgenden an ausgewählten Modellen, die diese Verbindung thematisieren, exemplarisch aufgezeigt. Brühwiler (2014) setzt sich am Beispiel der ,,adaptiven Lehrkompetenz “ mit der Thematik auseinander und entwirft ein Prozessmodell mit den Bereichen „Planungskompetenz“ und „Handlungskompetenz" (Abb. 2).

Den Hintergrund des Modells bildet die Sachkompetenz, definiert als fundiertes fachliches Wissen. Zur Planungskompetenz, die vor dem eigentlichen Unterrichten wirksam wird, gehört zum einen die diagnostische Kompetenz zum Erfassen der Lernvoraussetzungen für das Erreichen bestimmter Ziele, operationalisiert durch die Einschätzung, wie gut die Lernenden ein bestimmtes Lernziel erreicht haben, und zum anderen die didaktische Kompetenz hinsichtlich des Einsatzes geeigneter Unterrichtsmaßnahmen. Die Handlungskompetenz erfordert gemäß Brühwiler (2014) in der konkreten Unterrichtssituation Klassenführungskompetenz, didaktische Kompetenz hinsichtlich des Einsatzes geeigneter Unterrichtsmaßnahmen sowie diagnostische Kompetenz zur Überprüfung des Erreichens der gesetzten Ziele. Die Sachkompetenz wird als bedeutsam für die Stoff- und Aufgabenauswahl und die interaktiven Unterrichtsphasen und somit als zentral für die didaktische Kompetenz in den Bereichen Planen und Handeln betrachtet. Diagnostisches und didaktisches Handeln werden somit in einer engen Verbindung gesehen. Hinsichtlich der Planungskompetenz ist die diagnostische Kompetenz der didaktischen Kompetenz vorgeordnet.

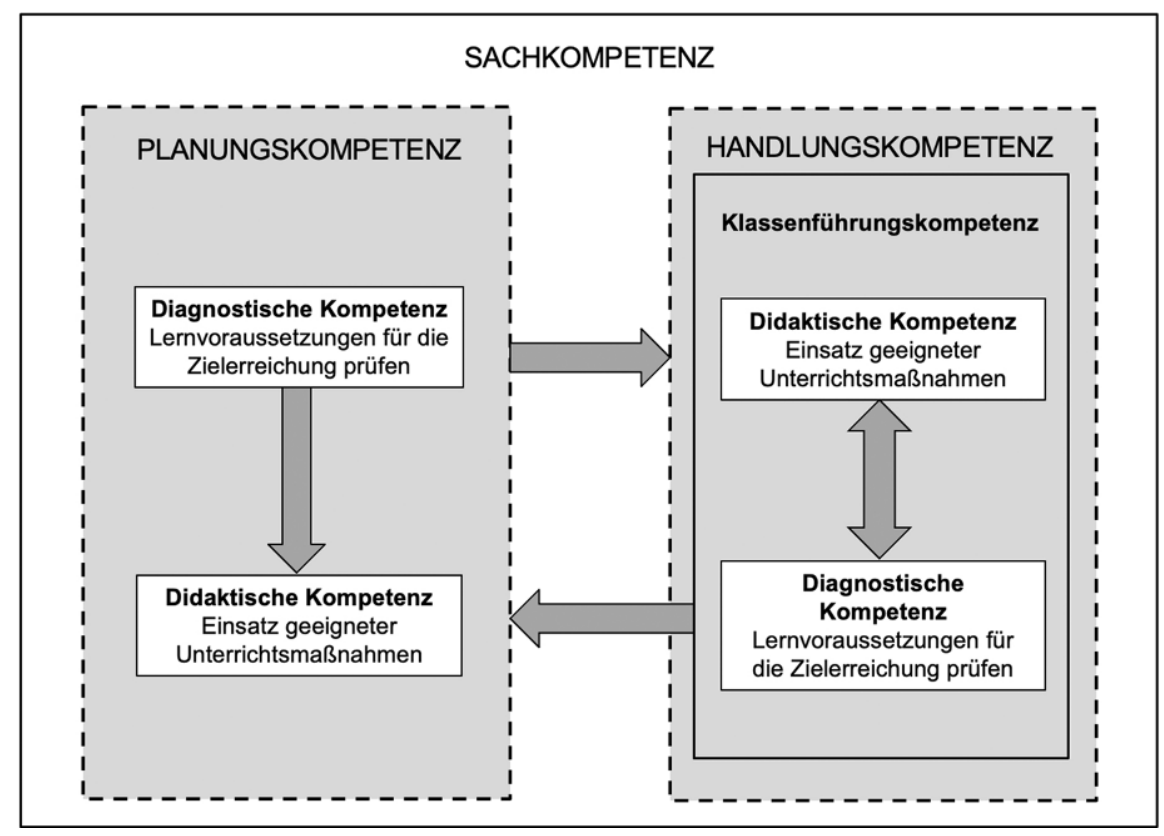

Abb. 2 Modell der adaptiven Lehrkompetenz. (Eigene Darstellung nach Brühwiler 2014, S. 92) 
Herppich et al. $(2017,2018)$ diskutieren die Verbindung von diagnostischem und didaktischem Handeln im Kontext eines Prozessmodells pädagogischer Diagnostik, das sich auf die Urteilsbildung bezieht. Diese wird als zentrales Qualitätsmerkmal des diagnostischen Handelns betrachtet. Die Autorinnen und Autoren trennen diagnostisches und didaktisches Handeln explizit. Begründet wird diese Trennung mit dem Argument, dass Diagnosen Informationen enthalten würden, die vor einer didaktischen Entscheidung gewonnen würden und deren Voraussetzung seien. Zentraler Bestandteil des Modells ist ein Flussdiagramm, das sich ausschließlich auf den Urteilsprozess bezieht. Endpunkt ist dabei ist immer die Diagnosestellung. Ausgehend von einem bestimmten Ziel werden in einem ersten Schritt diagnostische Informationen zu den Lernenden verarbeitet. Danach wird entschieden, ob allenfalls zusätzliche Informationen notwendig sind, und diese werden - wenn erforderlich in einem hypothesengeleiteten Prozess erhoben. Das diagnostische Handeln ist somit dem didaktischen Handeln vorgeordnet. Dabei gehen die Autorinnen und Autoren davon aus, dass diagnostisches und didaktisches Handeln zumindest teilweise andere Kompetenzen erfordern. Für das diagnostische Handeln wird „Assessement-Kompetenz" (Herppich et al. 2018) als bedeutsam erachtet. Sie umfasst die Kenntnis von Konzepten zur formativen (lernunterstützenden) und zur summativen (bewertenden) Beurteilung, Wissen zu verschiedenen Testmethoden (z. B. Testentwicklung, Testinterpretation) oder Wissen zu Beurteilungseffekten. Es wird auch darauf hingewiesen, dass inhaltsspezifische Aspekte zu berücksichtigen seien wie beispielsweise Wissen über häufige Fehlkonzepte zu bestimmten Lerninhalten. Darauf wird jedoch nicht näher eingegangen und es wird nicht ausgeführt, welche Rolle dieses Wissen im diagnostischen Prozess spielt.

\subsubsection{Pädagogische Diagnostik im Kontext der Mathematikdidaktik}

Die wissenschaftliche Auseinandersetzung mit dem diagnostischen und pädagogischen Handeln bezogen auf das mathematische Lernen ist stark von den Ansätzen der pädagogischen Diagnostik geprägt. Entsprechende Untersuchungen stammen zum Teil aus der pädagogischen Psychologie, zum Teil aus der Mathematikdidaktik und beziehen sich auf ganz unterschiedliche Themen. Analysiert wurde beispielsweise die Akkuratheit der Einschätzung der Leistungen von Schülerinnen und Schülern oder die Aufgabenschwierigkeit (z. B. Anders et al. 2010; Karst et al. 2014; Hoffmann und Böhme 2014), die Einschätzung der Produkte von Lernenden (z. B. Hoth et al. 2016; Philipp 2018) sowie der Umgang der Lehrkräfte mit Fehlern (Heinrichs und Kaiser 2018). Dabei steht häufig die Einschätzung der Akkuratheit der Urteile der Lehrkräfte im Mittelpunkt. Im DiaCoM-Modell (Explaining Teachers' Diagnostic Judgements by Cognitive Modeling; Leuders und Loibl 2021; Loibl et al. 2020) werden die kognitiven Prozesse, die zu einer Beurteilung oder Diagnose führen, analysiert. Diagnostisches Denken wird als Informationsverarbeitungsprozess modelliert, der von der Situation (z. B. Aufgabenspezifika, Schülerinnen und Schülern, Rahmenbedingungen wie Zeitdruck) und den Merkmalen der Person (Wissen, Beliefs, Motivation, aktuelle Befindlichkeiten wie Stress) beeinflusst wird. Anhand dieses Modells wurde beispielsweise untersucht, wie verschiedene Faktoren (z.B. das fachdidaktische Wissen, Aufgabenmerkmale, die für die Aufgabenbearbeitung 
verfügbare Zeit) das Urteilsverhalten beeinflussen (Rieu et al. 2020). Dabei wurde aufgezeigt, dass die Kurzvermittlung von spezifisch fachdidaktischem Wissen zu einer akkurateren Identifizierung und Gewichtung von schwierigkeitsgenerierenden Merkmalen mathematischer Aufgaben und somit zu einer höheren Urteilsgenauigkeit führte. Das heißt, dass spezifisch fachdidaktisches Wissen für den Prozess der Urteilsbildung eine zentrale Rolle zu spielen scheint. Nicht einbezogen wird in diesem Modell das didaktische Handeln.

\subsection{Diskussion der Ansätze}

\subsubsection{Aufgabenfelder}

Während sich die pädagogische Diagnostik auf ein breites Aufgabenfeld mit unterschiedlichen Zielsetzungen (Optimierung der individuellen Lernprozesse, Feststellung von Lernergebnissen; Ingenkamp 1985) bezieht, stehen bei der Förderdiagnostik immer die Optimierung der individuellen sowie oft auch beeinträchtigten Lernprozesse und geeignete Fördermaßnahmen im Zentrum. Dabei geht es nicht darum, die Leistungen von Lernenden in eine Reihenfolge zu bringen oder zu überprüfen, ob bestimmte Lernziele erreicht worden sind. Im Mittelpunkt steht immer eine differenzierte Erfassung der individuellen Kompetenzen der Schülerinnen und Schüler, das Auffinden von Schwierigkeiten und das Bereitstellen von geeigneten Fördermaßnahmen. Dies verfolgt in der Regel das Ziel, einen Förderplan zu erstellen, in dem die vorhandenen Kompetenzen und die angestrebten Ziele differenziert festgehalten werden (Popp et al. 2017). In einer typischen förderdiagnostischen Situation hat eine Lehrkraft beispielsweise festgestellt, dass ein Kind das Lernziel ,,verdoppeln, halbieren, addieren und subtrahieren im Zahlenraum bis 100“ nicht erreicht hat. In einer förderdiagnostischen Erfassung in der Einzelsituation wird es darum gehen, mit einer Auswahl von geeigneten Aufgaben und einem diagnostischen Interview den Lernstand des Kindes differenziert zu erfassen und zu überprüfen, welche Aufgaben das Kind lösen kann, ob es diese mit oder ohne Veranschaulichungen bearbeiten kann, ob allenfalls Vorkenntnisse fehlen, usw. Ein solches Vorgehen ist auch in der pädagogischen Diagnostik vorgesehen (z. B. Von Aufschnaiter et al. 2015), steht jedoch zumindest im aktuellen Diskurs im Hintergrund. Insgesamt sind die Zielsetzungen in vielen Ansätzen der pädagogischen Diagnostik im Vergleich zur Förderdiagnostik stärker auf die Sozialnorm und den Status und weniger auf die individuellen Lernprozesse ausgerichtet. Diese unterschiedlichen Aufgaben und Zielsetzungen von Förderdiagnostik und pädagogischer Diagnostik erfordern jeweils auch andere Vorgehensweisen, Methoden und Kompetenzen der Lehrkräfte. Während in der pädagogischen Diagnostik die Kenntnis von diagnostischen Verfahren, Gütekriterien, Testentwicklung, Testinterpretation sowie von Urteilsfehlern und -tendenzen als sehr wichtig betrachtet wird (Herppich et al. 2017, 2018; Helmke 2015) und somit entsprechende diagnostische Kompetenzen der Lehrkräfte eine zentrale Rolle spielen, wird in der Förderdiagnostik Gewicht auf Aspekte wie eine vertrauensfördernde Kommunikation (Popp et al. 2017) oder sogenannte ,qualitative Gütekriterien“ wie Übereinstimmung der Einschätzungen im Team gelegt (Eggert und Reichenbach 2007). Das heißt, dass - bedingt durch die unterschiedlichen Auf- 
gabenfelder - in der Förderdiagnostik und der pädagogischen Diagnostik andere Schwerpunkte bezüglich der Kompetenzen der Lehrkräfte gesetzt werden.

\subsubsection{Diagnostisches und didaktisches Handeln}

In der Förderdiagnostik ist eine enge, jedoch auch kritisch zu sehende Verbindung von diagnostischem und didaktischem Handeln konstitutiv, die mit entsprechenden Prozessmodellen (Abb. 1) dargestellt wird. Obwohl Schlee seit Jahrzehnten auf das Problem der Vermischung von Deskription und Präskription und die fehlenden Kriterien für die Urteilsbildung hinweist, wird diesem Einwand in den konkreten Vorschlägen zur Umsetzung der Förderplanung (z.B. Popp et al. 2017; Buholzer 2014) kaum Beachtung geschenkt.

Etwas anders präsentiert sich die Situation in der Mathematikdidaktik bzw. in auf das mathematische Lernen bezogenen Publikationen, die der Förderdiagnostik zugeordnet werden können. In diesen Veröffentlichungen (z. B. Prediger et al. 2017; Hußmann und Prediger 2014a, b; Kaufmann und Wessolowski 2006; Scherer 2018a, b, 2019) liegen Diagnoseaufgaben, Förderhinweise und konkrete Aufgaben vor. Diese basieren auf fachlichen und fachdidaktischen Grundlagen wie z. B. Grundvorstellungen, didaktischen Prinzipien oder fachlichen Analysen von konkreten mathematischen Inhaltsbereichen (z.B. dezimales Stellenwertsystem). Damit wird implizit zwischen Deskription und Präskription unterschieden. Konzeptuelle Überlegungen sind jedoch nicht das Ziel dieser Publikationen, sondern die Lehrkräfte sollen bei der Aufgabe des diagnostischen und didaktischen Handelns und damit auch bei der Interpretation der Beobachtungen und der Ergebnisse von Lernstandserfassungen unterstützt werden. In den Veröffentlichungen von Hößle et al. (2017), Moser Opitz und Nührenbörger (2015), Scherer und Moser Opitz (2010) sowie Wember (2018) wird die Problematik der Ableitung von Förderzielen aus der Diagnose kritisiert, und es wird auf die Bedeutung des fachdidaktischen Wissens für das diagnostische und didaktische Handeln hingewiesen. Allerdings erfolgt dies allgemein bezogen auf das diagnostische und didaktische Handeln, ohne dass zwischen diesen beiden Handlungsaspekten differenziert wird.

In der pädagogischen Diagnostik wurde die Diskussion zur Unterscheidung von Deskription und Präskription bisher nicht geführt, obschon die Urteilsbildung in vielen Modellen als zentral betrachtet und untersucht wird. Das heißt, dass eine wichtige erkenntnistheoretische Unterscheidung wenig beachtet worden ist. Zudem wird der Zusammenhang von diagnostischem und didaktischem Handeln je nach Modell unterschiedlich gesehen bzw. noch nicht umfassend thematisiert. Im Modell von Brühwiler (2014) besteht - wie bei der Förderdiagnostik - eine enge Verbindung zwischen diagnostischem und didaktischem Handeln und die Sachkompetenz bildet im Modell die Grundlage für die Planungs- und die Handlungskompetenz (Abb. 2). Allerdings wird die Bedeutung der Sachkompetenz nur bezogen auf das didaktische Handeln (Aufgabenauswahl, Beantworten der Fragen der Lernenden) ausdifferenziert und deren Rolle für das diagnostische Handeln wird nicht ausgeführt. Somit bleibt unklar, auf welcher Basis die Urteilsbildung erfolgt und wie die diagnostischen Informationen für den Einsatz geeigneter Unterrichtsmaßnahmen genutzt werden. Das heißt, dass sich die Problematik des Ableitens von didaktischen 
Maßnahmen aus Beobachtungen auch hier zu zeigen scheint. Im Konzept von Herppich et al. (2017, 2018) wird der diagnostische Prozess von Hypothesenbildung, Erheben von Informationen, erneuter Hypothesenbildung usw. mit einem Prozessmodell differenziert dargestellt und explizit vom didaktischen Handeln getrennt. Als wichtig für den Urteilsprozess erachtet werden spezifisch diagnostische Kompetenzen (z. B. Konzepte zu formativen und summativen Beurteilungen, Wissen zu verschiedenen Assessement-Methoden, Wissen zu Beurteilungseffekten). Fachliche und fachdidaktische Kompetenzen werden als Teil der diagnostischen Kompetenz aufgeführt, ohne dass geklärt wird, welche Rolle diese im Urteilsprozess spielen. Zudem fehlen Hinweise, anhand welcher Kriterien die Urteilsbildung und darauf aufbauend die didaktische Entscheidung erfolgen. Im aus der Mathematikdidaktik stammenden DiaCoM-Modell (Loibl et al. 2020; Rieu et al. 2020) wird im Gegensatz zu den beiden anderen referierten Modellen das fachdidaktische Wissen der Lehrkräfte als bedeutsam für das diagnostische Handeln erachtet. Allerdings wird hier die Verbindung zum didaktischen Handeln nicht näher ausgeführt, da im Modell die Informationsverarbeitung während des diagnostischen Prozesses im Vordergrund steht.

Zusammenfassend zeigt sich erstens, dass die Frage, auf welcher Grundlage die Urteilsbildung und die didaktische Entscheidung im diagnostischen Prozess erfolgen und somit die Frage nach der Unterscheidung von Deskription und Präskription weder in der Förderdiagnostik noch in der pädagogischen Diagnostik zufriedenstellend geklärt worden ist. Zudem wird zweitens der Zusammenhang von diagnostischem und didaktischem Handeln in den dargestellten Modellen unterschiedlich gesehen. In der Förderdiagnostik und im Modell von Brühwiler (2014) ist diese Verbindung eng, im Modell von Herppich et al. $(2017,2018)$ und im DiaCoM-Modell wird explizit eine Trennung vorgenommen. Drittens bestehen Unterschiede bezüglich der Bedeutung und Gewichtung der diagnostischen und didaktischen Kompetenzen für das diagnostische und didaktische Handeln. In der Förderdiagnostik werden diese nicht thematisiert. Bei Brühwiler (2014) steht die Bedeutung der Sachkompetenz für die Handlungskompetenz im Zentrum. Loibl et al. (2020) betonen, dass das fachdidaktische Wissen auch für das diagnostische Handeln wichtig ist und in der Studie von Rieu et al. (2020) wird dessen Bedeutung auch empirisch nachgewiesen. Bei Herppich et al. (2017, 2018) steht die Assessment-Kompetenz (z. B. Kenntnis von diagnostischen Verfahren, Testentwicklung, Testinterpretation, Urteilsfehlern und -tendenzen) bezüglich des diagnostischen Handelns im Vordergrund.

Im Folgenden werden ausgehend von den Arbeiten von Klauer (1973) und Morris et al. (2009) Überlegungen dargestellt, die dazu beitragen, die Lücken, die sich bezüglich der Verbindung von diagnostischem und didaktischem Handeln in den ausgewählten Modellen zeigen, zu schließen.

\section{Professionelles mathematikspezifisches Wissen als Grundlage für diagnostisches und pädagogisches Handeln}

Wenn es um diagnostisches und didaktisches Handeln geht, ist die Reflexion bezüglich des Verhältnisses von Deskription und Präskription wichtig. Um dieses zu 
analysieren, ist die von Klauer (1973) vorgenommene Unterscheidung zwischen deskriptiver, präskriptiver und normativer Pädagogik hilfreich. Klauer präzisiert damit die bisher allgemein verwendeten erkenntnistheoretischen Begriffe Deskription und Präskription. Diese begriffliche Klärung ist hilfreich, um die Verbindung von diagnostischem und didaktischem Handeln zu reflektieren, insbesondere hinsichtlich der Urteilsbildung und der Entscheidungsfindung, die in jedem diagnostischen Prozess bedeutsam sind. Die deskriptive Pädagogik erforscht nach Klauer (1973) die Realität der Erziehung, es geht darum, die Erziehungswirklichkeit (Lehr- und Lernziele, Organisationsformen, Persönlichkeitsmerkmale sowie Verhaltensweisen) $\mathrm{zu}$ beschreiben und (theoriegeleitet) zu interpretieren. Das diagnostische Handeln kann somit vereinfachend der deskriptiven Pädagogik zugeordnet werden. Bei der präskriptiven Pädagogik geht es um die eigentliche Aufgabe der Erziehung oder - wie Klauer (1973, S. 84) schreibt - um eine „Theorie des Änderns“. Diese ,stellt Änderungswissen bereit, ein Wissen, das Handlungsanweisungen ermöglicht“ (Klauer 1973, S. 85; Hervorhebung im Original). Es geht somit um eine Theorie des pädagogischen bzw. des didaktischen Handelns, auf deren Grundlage Erziehungswirklichkeit interpretiert werden kann. Klauer (ebd.) betont auch, dass die präskriptive Pädagogik weder die Voraussetzungen beschreiben kann - das ist die Aufgabe der deskriptiven Pädagogik - noch die Zielsetzungen festlegen kann. Letzteres ist Aufgabe der normativen Pädagogik, die darin besteht, übergreifende ,Sollvorstellungen" festzulegen und zu prüfen. Nach Ricken und Schuck (2011) und Sturm et al. (2019) gehören dazu das Menschenbild, die Vorstellungen von Lernen und menschlicher Entwicklung oder die Zielsetzungen diagnostischer Tätigkeiten. Fachbezogene Normen beziehen sich beispielsweise auf Curricula oder Bildungsstandards. Die normative Pädagogik wird in den folgenden Ausführungen vernachlässigt, da es sich dabei um eher allgemeine Zielsetzungen handelt, die im Diagnoseprozess eine untergeordnete Rolle spielen.

Wie dargelegt worden ist, weist Klauer (1973) dem Änderungswissen eine hohe Bedeutung für das pädagogische Handeln zu. Hier stellt sich die Frage, was dies für die Mathematikdidaktik konkret bedeutet. Ein Ansatz, der das Änderungswissen bezüglich des mathematischen Lernens beschreibt und explizit berücksichtigt, stammt von Morris et al. (2009). Die Autorinnen und Autoren orientieren sich am Kompetenzmodell von Ball et al. (2008). Sie heben die Bedeutung des ,,specialized content knowledge“, das im Modell von Ball et al. (2008) zum ,mathematical knowledge for teaching" gehört, hervor und bezeichnen dieses als Wissen, das spezifisch und essentiell ist, um Mathematik zu unterrichten. Dabei weisen sie darauf hin, dass es sich um eine besondere Form des von Shulman (1986) beschriebenen ,pedagogical content knowledge" handle und von diesem zu unterscheiden sei:

This kind of knowledge falls outside Shulman's pedagogical content knowledge because it does not draw exactly on knowledge on students or teaching. It is content knowledge, but content knowledge of a particular kind. It is implicated in common tasks such as choosing representations of mathematical ideas that reveal key subconcepts of the ideas, evaluating whether student responses show an understanding of key subconcepts, and justifying why arithmetic algorithms work (Morris et al. 2009, S. 494). 
Dieses mathematikspezifische Wissen bildet den Ausgangspunkt für beides, die Analyse von Lernprozessen (diagnostisches Handeln) und von Unterricht (didaktisches Handeln) und manifestiert sich im Spezifizieren von Lernzielen. Morris et al. (2009, S. 493) bezeichnen dies als „unpacking learning goals“ und illustrieren am Beispiel der Addition von Dezimalbrüchen, welche Überlegungen Lehrkräfte anstellen müssen, um Lernziele ,aufzuschlüsseln“ (ebd., S. 493):

1. A quantity is identified as the quantity „one.“

2. The value of each decimal number is determined by its place in the numeral. Each place is associated with a unit of measure, and the size of the units increase or decrease by a factor of 10 as you move to the left or right, respectively.

3. When adding decimals, same-sized units are joined, and the sum will be of that unit.

4. When a quantity contains 10 or more of a particular unit of measure, those 10 can be exchanged for one unit of measure of the next larger size.

Die Autorinnen und Autoren weisen darauf hin, dass es sich hier um spezifisches Fachwissen handle, das auch Wissen zu geeigneten Repräsentationen von mathematischen Ideen und zu Subkonzepten sowie die Einschätzung, ob die Lernenden die entsprechenden Konzepte verstanden haben, beinhalten würde. Dieses spezifische Wissen zum Unterrichten von Mathematik wird hier somit nicht nur für das didaktische Handeln, sondern auch als Voraussetzung für die Erfassung des Lernstandes und somit für das diagnostische Handeln als wichtig erachtet. Nur wenn eine Lehrkraft den Lerngegenstand sehr gut kennt, weiß sie, worauf sie in der diagnostischen Situation achten muss, kann sie Schwierigkeiten erkennen. Das heißt, dass dieses Wissen als Änderungswissen notwendig ist um diagnostisch und didaktisch handeln zu können. Das folgende Beispiel aus einer eigenen Untersuchung kann die Bedeutung des Ansatzes von Morris et al. (2009) illustrieren.

Um zu überprüfen, ob es Grundschullehrkräften und förderpädagogischen Lehrkräften gelingt, auf der Grundlage ihres fachlichen und fachdidaktischen Wissens zu einer fehlerhaften Aufgabenbearbeitung eines Kindes weitere Diagnoseaufgaben zu stellen, wurde ihnen eine Fallvignette (Abb. 3) mit folgender Aufgabe vorgelegt. Die Abbildung zeigt Divisionsaufgaben, die ein Kind mit einer Rechenschwäche im

Abb. 3 Fallvignette für Lehrkräfte zur Entwicklung einer diagnostischen Aufgabe. (Eigene Abbildung)

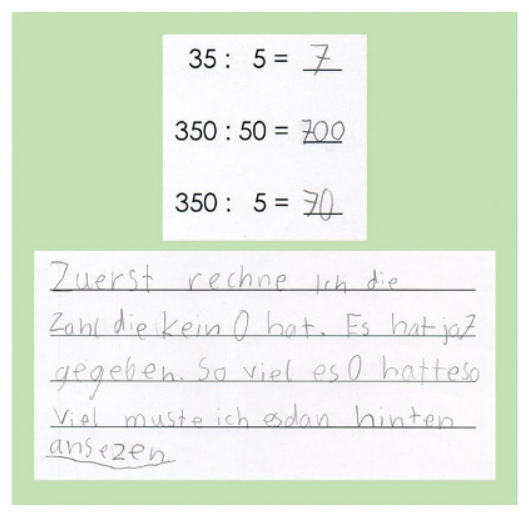


vierten Schuljahr teilweise falsch gelöst hat. Welche Aufgabe würden Sie dem Kind stellen, um weitere Informationen zu seinem Lernstand zu erhalten?

Die falsch gelöste Aufgabe 350: 50=700 kann auf verschiedene Verstehenslücken hinweisen, und es gibt verschiedene Möglichkeiten für geeignete weiterführende diagnostische Aufgaben. Da der Quotient in der falschen Lösung größer ist als der Dividend könnte dies darauf hinweisen, dass das Konzept der Division noch nicht gefestigt ist. Die Erklärung des Kindes gibt aber auch Anlass zur Vermutung, dass Aspekte des Dezimalsystems noch nicht hinreichend verstanden sind. Es könnte auch sein, dass das Prinzip des Multiplizierens mit Stufenzahlen übergeneralisiert und auf die Division übertragen wird. Das heißt, dass - je nach Hypothese - unterschiedliche weitere Diagnoseaufgaben gestellt werden können und auch sinnvoll sind. Das erfordert jedoch, dass Lehrkräfte im Sinne des Aufschlüsselns von Lernzielen (Morris et al. 2009) auf der Grundlage ihres fachdidaktischen Wissens Hypothesen aufstellen und davon ausgehend mögliche diagnostische Aufgaben formulieren können. Exemplarische Antwortbeispiele (Tab. 1) zeigen, dass dies Lehrkräften in unterschiedlichem Maß gelingt. Während einige der vorgeschlagenen Diagnoseaufgaben darauf abzielen, durch das Bearbeiten der Umkehraufgabe oder der Multiplikation von Stufenzahlen (Antwortbeispiele 1 und 2) zusätzliche Informationen über den Lernstand des Kindes und dessen Verständnis zu gewinnen, wird im Beispiel 3 keine Diagnoseaufgabe entwickelt, sondern eine rezepthafte Erklärung vorgeschlagen. Auch hinsichtlich des Einsatzes von Veranschaulichungen werden Unterschiede in der didaktischen Reflexion der antwortenden Personen deutlich. Während ein geeigneter Vorschlag lautet, die Aufgabe mit Arbeitsmitteln, die eine dezimale Struktur beinhalten, zu bearbeiten (Antwortbeispiel 4), enthält die Antwort 5 einen sehr unspezifischen Hinweis auf den Einsatz von Arbeitsmitteln, und zwar sowohl bezüglich des mathematischen Inhalts (,Malrechnungen“) als auch des konkreten Materials (,wo die Menge deutlich wird“").

Die Antworten der Lehrkräfte in Tab. 1 macht deutlich, dass das von Morris et al. (2009) beschriebene mathematikspezifische fachliche Wissen als Änderungs-

Tab. 1 Antwortbeispiele für weiterführende diagnostische Aufgaben zur falsch gelösten Rechnung $350: 50=700$

\begin{tabular}{lll}
\hline & Thematischer Fokus & Antwortbeispiel \\
\hline 1 & $\begin{array}{l}\text { Multiplizieren mit Stufenzah- } \\
\text { len }\end{array}$ & $\begin{array}{l}\text { Ähnliche Aufgaben zur Multiplikation. Also z. B. } 7 \cdot 5,7 \cdot 50, \\
70 \cdot 50\end{array}$ \\
2 & Umkehraufgabe nutzen & $\begin{array}{l}\text { Ich würde die entsprechenden Aufgaben zur Multiplikation } \\
\text { stellen, um herauszufinden, ob es diese verstanden hat, um dann } \\
\text { daraus die Division abzuleiten }\end{array}$ \\
3 & $\begin{array}{l}\text { Rezept „Null anhängen, weg- } \\
\text { lassen“ }\end{array}$ & $\begin{array}{l}\text { Ich würde dem Kind erklären, dass wenn es auf der anderen } \\
\text { Seite auch eine 0 hat von der Division, dass man beide streichen } \\
\text { kann und man diese nicht mehr am Schluss anhängen muss } \\
\text { Die gleichen Aufgaben mit Dienes-Material lösen }\end{array}$ \\
$\begin{array}{l}\text { Arbeitsmittel/Veranschaulich- } \\
\text { ungen mit Fokus auf dezimale } \\
\text { Struktur nutzen } \\
\text { Arbeitsmittel/Veranschaulich- } \\
\text { ungen ohne dezimale Struktur } \\
\text { nutzen }\end{array}$ & $\begin{array}{l}\text { Ich würde Malrechnungen einbeziehen und Anschauungsmateri- } \\
\text { al, wo die Menge deutlich wird, verwenden }\end{array}$ \\
\hline
\end{tabular}


wissen nicht nur für das didaktische, sondern auch für das diagnostische Handeln bedeutsam ist. Nur wenn Wissen über einen bestimmten Lerngegenstand vorhanden ist, können passende Diagnoseaufgaben gestellt und die Antworten und Lösungen der Schülerinnen und Schülern interpretiert werden. Der Ansatz von Morris bietet somit wichtige Hinweise zur Beantwortung der Frage, auf welcher Grundlage diagnostische Informationen beurteilt und didaktische Entscheidungen getroffen werden können. Allerdings wird dabei die in anderen Modellen wichtige Assessment-Kompetenz nicht berücksichtigt. Zudem fehlt das für das didaktische Handeln wichtige „mathematical knowledge for teaching“ (Ball et al. 2008). Dazu gehört beispielsweise Wissen zu geeigneten Veranschaulichungen oder Wissen zu Vor- und Nachteilen von verschiedenen Rechenverfahren. Das heißt, dass der Ansatz von Morris et al. (2009) erweitert werden muss, um die Verbindung von diagnostischem und didaktischem Handeln und dem dafür notwendigen Professionswissen zu modellieren.

\section{Diagnostisches Handeln und didaktisches Handeln verbinden: ein Prozessmodell}

Die bisherigen Ausführungen haben erstens gezeigt, dass in der pädagogischen Diagnostik und in der Förderdiagnostik unterschiedliche Aufgabenfelder in den Blick genommen werden. Das führt zu unterschiedlichen Ziel- und Schwerpunktsetzungen und hat auch Folgen für die Frage nach den professionellen (diagnostischen und didaktischen) Kompetenzen der Lehrkräfte. Das Herstellen einer Rangreihenfolge der Schülerinnen und Schüler nach Leistung stellt andere Anforderungen als die Analyse der Aufgabenbearbeitung einer Schülerin oder eines Schülers mit mathematischen Lernschwierigkeiten, die Durchführung und Interpretation einer Lernstandserfassung sowie eine darauf abgestimmte Förderplanung. Das heißt, dass hinsichtlich des Aufbaus von entsprechenden Kompetenzen der Lehrkräfte die unterschiedlichen Aufgabenfelder und Zielsetzungen berücksichtigt werden müssen (Von Aufschnaiter et al. 2015). Diese Thematik wurde im wissenschaftlichen Diskurs bisher noch wenig behandelt, da meistens von einem globalen Konstrukt der diagnostischen (bzw. didaktischen) Kompetenzen ausgegangen wird. In diesem Zusammenhang stellt sich die Frage nach der Verwendung des Begriffs „Kompetenzen“. In den Ansätzen der pädagogischen Diagnostik (z.B. Brühwiler 2014; Helmke 2015; Herppich et al. 2017, 2018) wird übergeordnet der Begriff der diagnostischen (und didaktischen) Kompetenz verwendet. Herppich et al. (2017) definieren Kompetenzen als stabile Dispositionen, die das Verhalten von Personen in einer bestimmten domänen-spezifischen Situation bestimmen. Beim von Morris et al. (2009) beschriebenen „specialized content knowledge" steht jedoch das Professionswissen im Vordergrund. Im Folgenden wird deshalb - ohne die Diskussion zum Zusammenhang von Wissen und Kompetenzen zu führen - in Anlehnung an den englischen Begriff ,knowledge“ der Begriff „Professionswissen“ verwendet. Durch die Verwendung des Begriffs „Wissen" wird auch betont, dass es im Prozess der diagnostischen Urteilsbildung und der darauf abgestimmten didaktischen Entscheidung um eine "Theorie des Änderns“ (Klauer 1973) geht. Zweitens wurde dargelegt, dass in den vorliegenden Konzepten nicht hinreichend geklärt worden ist, wie diagnostisches und didaktisches Handeln 
miteinander in Verbindung stehen (2.3.2). Damit verbunden bleibt drittens unklar auf welcher Grundlage die Urteilsbildung und die didaktische Entscheidung erfolgen. Gemäß Klauer (1973) ist dafür Änderungswissen zentral. Bezogen auf das mathematische Lernen haben Morris et al. (2009) aufgezeigt, dass spezialisiertes, mathematikbezogenes Professionswissen diese Funktion übernehmen und als Grundlage für die Urteilsbildung dienen kann. Allerdings reicht - wie dargelegt worden ist dieses Änderungswissen nicht aus für die Verbindung von diagnostischem und didaktischem Handeln. Um zur Schließung dieser Forschungslücken beizutragen wird im Folgenden (Abb. 4) ein Modell vorgestellt, das wichtige Erkenntnisse aus den vorangehend diskutierten Modellen und Konzepten integriert und aufzeigt, welche professionellen Wissensbestände in welcher Phase des Prozesses von diagnostischem und didaktischem Handeln zentral sind und auch grundlegend sind für die Urteilsbildung und die didaktische Entscheidung.

Im Mittelpunkt des Modells (Abb. 4) steht - analog dem Kreismodell der Förderdiagnostik (Abb. 1) und dem Modell von Brühwiler (2014; Abb. 2) - der zirkuläre Prozess des diagnostischen und didaktischen Handelns, verbunden durch die Urteilsbildung und die didaktische Entscheidung. Den Hintergrund (graue Fläche) bilden die verschiedenen Aufgabenfelder des diagnostischen und didaktischen Handelns. Die am Rand der grauen Fläche aufgeführten Beispiele können beliebig erweitert werden.

Das diagnostische Handeln beinhaltet das Erfassen der Lernvoraussetzungen der Schülerinnen und Schüler. Das didaktische Handeln umfasst die Planung - und

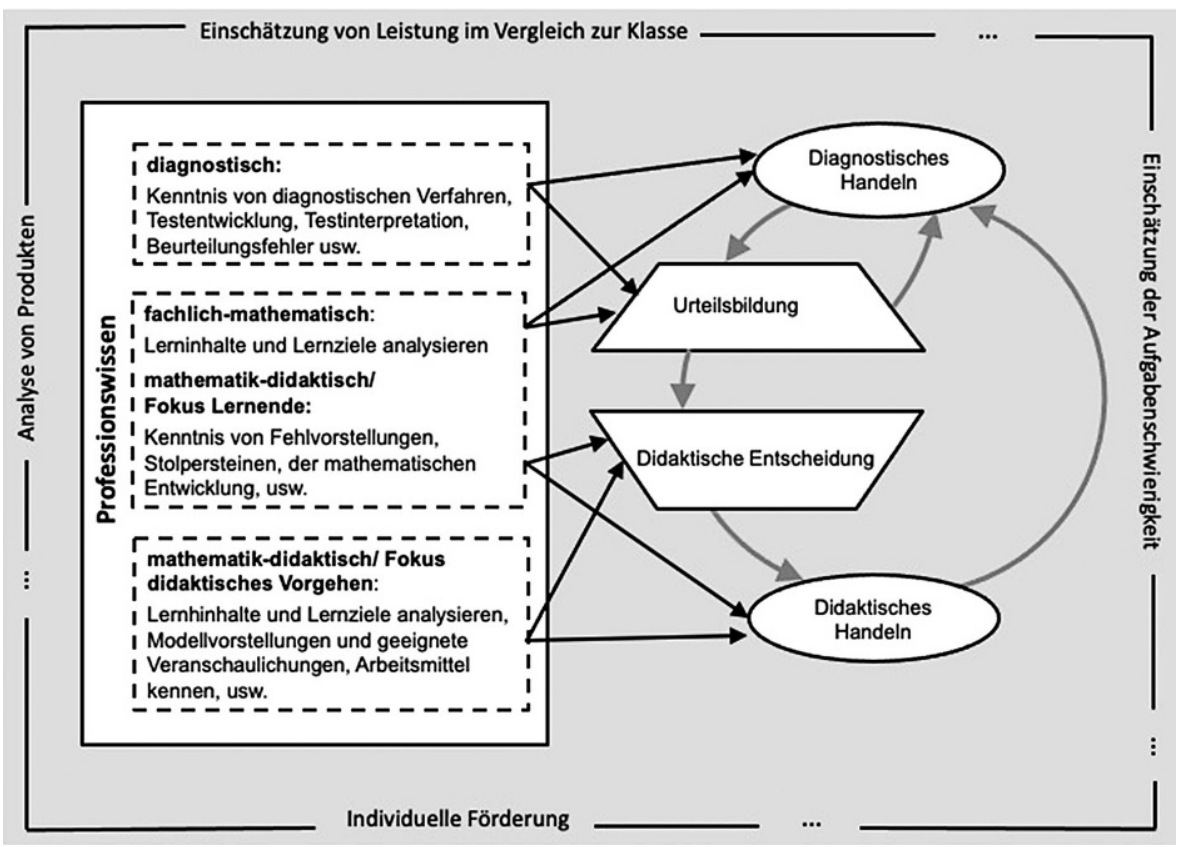

Abb. 4 Prozessmodell zur Verbindung von diagnostischem und didaktischem Handeln in der Mathematikdidaktik 
spätere Durchführung - der unterrichtlichen Maßnahmen, die abgestimmt auf das Ergebnis der Diagnose vollzogen werden. In der konkreten Umsetzung ist das diagnostische Handeln der Ausgangspunkt und dem didaktischen Handeln zeitlich vorgeordnet. Diagnostisches Handeln findet in der Regel jedoch nicht einmalig statt, sondern ist - wie bei Herppich et al. $(2017,2018)$ differenziert beschrieben haben ein zirkulärer Prozess von Informationsgewinnung, Hypothesenbildung, allenfalls erneuter Informationsgewinnung usw. Dies wird in der Grafik mit dem zusätzlichen Pfeil zwischen diagnostischem Handeln und Urteilsbildung dargestellt.

Zwischen dem diagnostischen und dem didaktischen Handeln findet der Prozess der Urteilsbildung und der didaktischen Entscheidung statt. Diese stehen in enger Verbindung, müssen jedoch auch getrennt betrachtet werden, da teilweise anderes Professionswissen notwendig ist. Links in der Grafik sind die unterschiedlichen Bereiche des Professionswissens dargestellt, die notwendig sind zur Auswahl der diagnostischen Informationen, zu deren Bewertung und Interpretation sowie zur Planung des didaktischen Handelns. Dabei stehen in den unterschiedlichen Phasen des Prozesses jeweils unterschiedliche Wissensbestände bzw. unterschiedliche Theorien des Änderns im Vordergrund.

Für das diagnostische Handeln ist einerseits spezifisch diagnostisches Professionswissen im Sinne der von verschiedenen Autorinnen und Autoren (z. B. Helmke 2015; Herppich et al. 2018; Loibl et al. 2020) beschriebenen Assessment-Kompetenz notwendig. Das reicht jedoch nicht aus, um diagnostische Informationen zu den Lernenden zu erheben, es ist auch fachspezifisches Wissen notwendig, um überhaupt zu entscheiden, welche Informationen erhoben werden sollen bzw. wichtig sind. Nach Morris et al. (2009) ist mathematikspezifisches Professionswissen im Sinne des ,unpacking learning goals“ (ebd.) wesentlich, im Modell als ,fachlich-mathematisches Professionswissen“ benannt. Zusätzlich ist jedoch auch Wissen nötig, wie es von Ball et al. (2008, S. 401) als ,knowledge of content and students“ operationalisiert worden ist, hier bezeichnet als ,mathematikdidaktisches Wissen mit Fokus auf die Lernenden“. Dazu gehört insbesondere Wissen zu Stolpersteinen, zu häufigen Fehlvorstellungen von Schülerinnen und Schülern, aber auch Wissen zur mathematischen Entwicklung, beispielsweise zur Zähl- oder Zahlbegriffsentwicklung. Diese Wissensbestände bilden zusammen mit dem diagnostischen Professionswissen im Sinne der präskriptiven Pädagogik auch die Grundlage für die theoriegeleitete Urteilsbildung bzw. für die Interpretation der erhobenen diagnostischen Informationen. Das eben beschriebene mathematikspezifische Professionswissen ist auch im nächsten Schritt, bei der didaktischen Entscheidung, wichtig. Für diese ist jedoch zusätzliches Professionswissen zu geeigneten didaktischen Vorgehensweisen in der Unterrichtssituation erforderlich. In der Terminologie von Ball et al. (2008, S. 401) handelt es sich um „knowledge of content and teaching“. Ein Beispiel soll dies veranschaulichen: Während zum Verständnis von Anteilen von Brüchen zusätzlich zum Rechteckmodell auch das Kreismodell eingesetzt werden kann, ist dieses zum Vergleichen von Prozenten und Brüchen eher nicht geeignet, da sich solche Vergleiche am Kreismodell nur schwer darstellen lassen. Prozente und Brüche lassen sich viel anschaulicher mit Bruchstreifen oder der Streifentafel vergleichen (Hußmann und Prediger 2014b). Bezüglich des didaktischen Handelns ist noch ein weiterer Bereich relevant, der im Modell nicht abgebildet wird, da er nicht im engeren Sinn 
zur Verbindung von diagnostischem und didaktischen Handeln gehört. Für die konkrete Umsetzung des didaktischen Handelns sind auch allgemeine pädagogische Kompetenzen $^{3}$ (Kunter et al. 2013) wie die Lernunterstützung (z.B. das Erteilen von lernförderlichem Feedback), das Stellen von kognitiv aktivierenden Fragen sowie eine erfolgreiche Klassenführung bedeutsam (Brühwiler 2014).

Je nach Aufgabenfeld sind jeweils andere Wissensbereiche bedeutsam. Wenn es um die Einschätzung von Lernenden im Vergleich zur Klasse geht, wenn die Leistungen der Schülerinnen und Schüler einer Klasse eine Reihenfolge gebracht werden oder wenn mit einem Kind ein standardisierter Test durchgeführt wird, um zu überprüfen, ob eine Rechenschwäche vorliegt, sind das diagnostische Professionswissen und die Kenntnis des mathematischen Lerngegenstands zentral. Wenn es um die Einschätzung der Schwierigkeit von Aufgaben geht, dann nimmt die Bedeutung des diagnostischen Professionswissens ab und fachliches und fachdidaktisches Wissen auf unterschiedlichen Ebenen werden wichtig.

Mit dieser Darstellung des komplexen Zusammenhangs von diagnostischem und didaktischem Handeln zeigt sich auch, dass die Frage, ob die Diagnostik der Didaktik vorgeordnet ist oder ob - wie es Schlee (1985a) formuliert hat - die Didaktik der Diagnostik vorgeordnet ist - je nach Perspektive unterschiedlich beantwortet werden kann. Hinsichtlich einer zeitlichen Abfolge ist - auch wenn der Prozess grundsätzlich zirkulär ist - das diagnostische Handeln der Urteilsbildung, der didaktischen Entscheidung und dem didaktischen Handeln vorgeordnet. Erst wenn eine Beobachtung oder die Einschätzung des Lernstandes erfolgt ist, kann eine didaktische Entscheidung folgen. Inhaltlich gesehen ist jedoch die Didaktik als Änderungswissen der Diagnostik vorgeordnet, da dieses notwendig ist, um den Gegenstand der Diagnose zu erkennen, eine Bewertung der diagnostischen Informationen vorzunehmen und die didaktische Entscheidung zu treffen.

\section{Fazit}

In den vorangehenden Ausführungen wurde aufgezeigt, dass die Auseinandersetzung zum Thema Diagnostik in der Mathematikdidaktik sowohl auf der pädagogischen Diagnostik als auch auf der Förderdiagnostik beruht, dass aber die zwei unterschiedlichen Zugänge oft unverbunden nebeneinanderstehen. Zudem wurde dargelegt, dass das diagnostische und didaktische Handeln in der Förderdiagnostik und den verschiedenen beschriebenen Modellen aus der pädagogischen Diagnostik und der Mathematikdidaktik unterschiedlich konzeptualisiert wird. Ein Modell zur Verbindung der beiden Handlungsbereiche fehlt. Das hier vorgestellte Modell beschreibt den Zusammenhang zwischen diagnostischem und didaktischem Handeln, indem zusätzlich zu diesen Handlungsebenen zwischen Urteilsbildung und didaktischer Entscheidung unterschieden wird. Ausgehend vom Konzept der deskriptiven und präskriptiven Pädagogik von Klauer (1973) wird aufgezeigt, dass Änderungswissen notwendig

\footnotetext{
${ }^{3}$ Hier wird in Abgrenzung zum theoriebasierten professionellen Wissen, das für die Beurteilung der diagnostischen Informationen und der didaktischen Entscheidung notwendig ist, explizit der Begriff „Kompetenz" verwendet.
} 
ist, um Handlungsanweisungen geben zu können und dass für die unterschiedlichen Phasen im Prozess des diagnostischen und didaktischen Handelns unterschiedliches professionelles Wissen bedeutsam ist.

Insbesondere wurde herausgearbeitet, dass - um einen logischen Fehlschluss zu vermeiden - die Bewertung der erhobenen diagnostischen Daten auf der Grundlage von fachlichen und fachdidaktischen Theorien bzw. vom entsprechenden professionellen Wissen der Lehrkräfte erfolgen muss. Darauf wird in verschiedenen aus der förderdiagnostischen Tradition stammenden mathematikdidaktischen Publikationen (Hößle et al. 2017; Moser Opitz und Nührenbörger 2015; Scherer und Moser Opitz 2010; Wember 2018) hingewiesen. In den praxisbezogenen Veröffentlichungen aus der Mathematikdidaktik wird dies umgesetzt, jedoch nicht konzeptualisiert. Bisher wurde jedoch in diesen Publikationen nicht aufgearbeitet, welche Wissensbereiche im Modell der Förderdiagnostik in welcher Phase des diagnostischen und didaktischen Handelns welche Bedeutung haben. Bezogen auf die Modelle aus der pädagogischen Diagnostik (Brühwiler 2014; Herppich et al. 2017, 2018) wurde erstens aufgezeigt, dass die Unterscheidung von Deskription und Präskription in den Modellen aus der pädagogischen Diagnostik nicht diskutiert worden ist. Zweitens wurde herausgearbeitet, dass neben der Assessment-Kompetenz auch das fachspezifische Professionswissen einen wichtigen Teil der diagnostischen Kompetenz darstellt, schon in der Phase der Erhebung der Informationen relevant ist und eine wichtige Grundlage für die Urteilsbildung und die didaktische Entscheidung darstellt. Dies wird jedoch - mit Ausnahme des DiaCoM-Modells - nicht berücksichtigt. In diesem spielen fachdidaktische Kompetenzen auch für das diagnostische Handeln eine zentrale Rolle, allerdings, ohne dass der Bezug zum didaktischen Handeln hergestellt wird. Morris et al. (2009) haben schließlich mit ihrem Vorschlag zum Aufschlüsseln von Lernzielen und dem damit verbundenen professionellen Wissen einen wesentlichen Beitrag zur Verbindung von diagnostischem und didaktischem Handeln geliefert, nehmen jedoch weitere Bereiche des Professionswissens wie z. B. die Assessment-Kompetenz nicht in den Blick. Mit dem hier vorgestellten Modell wird beabsichtigt, einen Beitrag zur Schließung der genannten Lücken zu liefern und insbesondere die Rolle des mathematikbezogenen Professionswissens hinsichtlich der Urteilsbildung und der didaktischen Entscheidung zu klären sowie herauszuarbeiten, in welchen Phasen des Prozesses von diagnostischem und didaktischem Handeln welche Wissensbestände zentral sind bzw. Schließlich wurde aufgezeigt, dass für unterschiedliche Zielsetzungen und Aufgabenfelder unterschiedliches Professionswissen der Lehrkräfte notwendig ist.

Die hier dargelegten Überlegungen und damit verbunden das Modell beinhalten auch Limitationen. Erstens wurden nur exemplarisch Modelle und Konzepte aus der pädagogischen Diagnostik und auch der Mathematikdidaktik ausgewählt und hinsichtlich der hier interessierenden Fragestellung analysiert. Eine andere Auswahl oder die Berücksichtigung von weiteren Konzepten könnten zu einer Ausdifferenzierung des Modells führen. Zweitens wurde der Begriff des „Professionswissen“ als Arbeitsbegriff eingeführt, ohne dass die Abgrenzung zum Begriff der diagnostischen und didaktischen Kompetenz diskutiert worden ist. Das würde den Rahmen des Artikels sprengen und müsste in einer weiteren Arbeit erfolgen. Drittens wurde der Begriff des mathematikspezifischen Professionswissens beschreibend von 
Morris et al. (2009) übernommen. Hier müsste eine vertiefte Auseinandersetzung auch bezüglich der Unterscheidung von fachlichem und fachdidaktischem Wissen stattfinden, wie sie beispielsweise von Prediger (2020) skizziert worden ist.

Aus diesen Überlegungen ergeben sich Folgerungen für drei Bereiche: die konzeptuelle Weiterentwicklung von Modellen zum diagnostischen und didaktischen Handeln, die Forschung sowie die Ausbildung von Lehrkräften. Bezüglich der konzeptuellen Entwicklung scheinen erstens im aktuellen Diskurs über die diagnostischen Kompetenzen von Lehrkräften die Thematik der Urteilsbildung und damit verbunden die Bedeutung des professionellen Wissens als Grundlage für die Interpretation der diagnostischen Informationen vernachlässigt worden zu sein. Dieser Thematik müsste - insbesondere hinsichtlich der Unterscheidung von deskriptiver und präskriptiver Pädagogik und dem damit verbundenen professionellen Wissen mehr Aufmerksamkeit geschenkt werden. In diesem Zusammenhang muss auch das hier entworfene Modell kritisch diskutiert und weiterentwickelt werden.

Zweitens ergeben sich Forschungsdesiderate. Es wäre beispielsweise vor dem Hintergrund der Studie von Rieu et al. (2020) interessant zu untersuchen, ob und wie sich das mathematikspezifische Professionswissen auf das Erfassen der Lernvoraussetzungen der Schülerinnen und Schüler, die Qualität der Diagnose und die Passung der didaktischen Entscheidung auswirkt. Das heißt auch, dass in der Forschung stärker differenziert werden müsste zwischen den im Modell dargestellten Bereichen des Professionswissen in den verschiedenen Phasen des diagnostischen Prozesses.

Für die Aus- und Fortbildung bedeuten die hier dargelegten Überlegungen drittens, dass die unterschiedlichen Phasen des zirkulären Prozesses von diagnostischem und didaktischem Handeln und die dafür notwendigen Bereiche professionellen Wissens berücksichtigt und explizit aufgearbeitet werden müssen. Lehrkräfte müssen ein Bewusstsein für die Unterscheidung zwischen deskriptiver und präskriptiver Pädagogik entwickeln und gezielt angeleitet werden, diagnostische Informationen theoriegeleitet zu interpretieren und entsprechende didaktische Entscheidungen zu treffen. In diesem Zusammenhang sind auch die unterschiedlichen Aufgabenfelder, die sich im Kontext von Unterricht ergeben, verstärkt zu beachten. Das heißt, dass vermehrt die Frage gestellt werden muss, welches Professionswissen für welche Aufgaben in erster Linie erforderlich sind. Das kann sich beispielsweise auch je nach Schulstufe unterscheiden. Wenn Übertrittsentscheide anstehen, rückt spezifisch diagnostisches Professionswissen stärker in den Vordergrund, wenn es um die individuelle Förderung eines Kindes mit Rechenschwäche geht, dann steht fachlich-mathematisches Professionswissen mit seinen unterschiedlichen Facetten im Vordergrund.

Es sind weitere konzeptuelle Überlegungen sowie empirische Studien zur Verbindung von diagnostischem und didaktischem Handeln notwendig, um die zentrale Forderung nach individueller Förderung gewährleisten zu können.

Funding Open access funding provided by University of Zurich

Open Access Dieser Artikel wird unter der Creative Commons Namensnennung 4.0 International Lizenz veröffentlicht, welche die Nutzung, Vervielfältigung, Bearbeitung, Verbreitung und Wiedergabe in jeglichem Medium und Format erlaubt, sofern Sie den/die ursprünglichen Autor(en) und die Quelle ord- 
nungsgemäß nennen, einen Link zur Creative Commons Lizenz beifügen und angeben, ob Änderungen vorgenommen wurden.

Die in diesem Artikel enthaltenen Bilder und sonstiges Drittmaterial unterliegen ebenfalls der genannten Creative Commons Lizenz, sofern sich aus der Abbildungslegende nichts anderes ergibt. Sofern das betreffende Material nicht unter der genannten Creative Commons Lizenz steht und die betreffende Handlung nicht nach gesetzlichen Vorschriften erlaubt ist, ist für die oben aufgeführten Weiterverwendungen des Materials die Einwilligung des jeweiligen Rechteinhabers einzuholen.

Weitere Details zur Lizenz entnehmen Sie bitte der Lizenzinformation auf http://creativecommons.org/ licenses/by/4.0/deed.de.

\section{Literatur}

Anders, Y., Kunter, M., Brunner, M., Krauss, S., \& Baumert, J. (2010). Diagnostische Fähigkeiten von Mathematiklehrkräften und ihre Auswirkungen auf die Leistungen ihrer Schülerinnen und Schüler. Psychologie in Erziehung und Unterricht, 57, 175-193. https://doi.org/10.2378/peu2010.art13d.

Ball, D. L., Thames, M.H., \& Phelps, G. (2008). Content knowledge for teaching: what makes it special? Journal of Teacher Education, 59, 389-407. https://doi.org/10.1177/0022487108324554.

Breitenbach, E. (2020). Förderdiagnostik - ein oft kritisiertes und problematisches, aber dennoch aktuelles Konzept in Pädagogik und Sonderpädagogik. Sonderpädagogische Förderung heute, 65(2), 171-182. https://doi.org/10.3262/SZ2002171.

Brühwiler, C. (2014). Adaptive Lehrkompetenz und schulisches Lernen. Münster: Waxmann.

Buholzer, A. (2014). Von der Diagnose zur Förderung. Grundlagen für den integrativen Unterricht. Zug: Klett.

Bundschuh, K. (1985). Dimensionen der Förderdiagnostik bei Kindern mit Lern-, Verhaltens- und Entwicklungsproblemen. München: Reinhardt.

Eggert, D., \& Reichenbach, C. (2007). Von den Stärken ausgehen. Individuelle Entwicklungspläne (IEP) in der Lernförderdiagnostik: Ein Plädoyer für andere Denkgewohnheiten und eine veränderte Praxis. Dortmund: Borgmann.

Fricke, S., \& Streit-Lehmann, J. (2015). Zum Einsatz von Entwicklungsplänen im inklusiven Mathematikunterricht. In A. Peter-Koop, T. Rottmann \& M. M. Lüken (Hrsg.), Inklusiver Mathematikunterricht in der Grundschule (S. 168-180). Offenburg: Mildenberger.

Gerich, M., Trittel, M., Bruder, S., Klug, J., Hertel, S., Bruder, R., \& Schmitz, B. (2017). Modeling, measuring, and training teachers' counseling and diagnostic competencies. In D. Leutner, J. Fleischer, J. Grünkorn \& E. Klieme (Hrsg.), Competence assessment in education: research, models and instruments (S. 149-166). Cham: Springer.

Heinrichs, H., \& Kaiser, G. (2018). Diagnostic competence for dealing with students' errors: fostering diagnostic competence in error situations. In T. Leuders, K. Philipp \& J. J. Leuders (Hrsg.), Diagnostic competence of mathematics teachers. Unpacking a complex construct in teacher education and teacher practice. Mathematics Teacher Education, (Bd. 11, S. 79-94). Cham: Springer. https://doi.org/ 10.1007/978-3-319-66327-2_4.

Helmke, A. (2015). Unterrichtsqualität und Lehrerprofessionalität. Diagnose, Evaluation und Verbesserung (6. Aufl.). Leipzig: Klett.

Helmke, A., \& Schrader, W. (1987). Interactional effects of instructional quality and teacher judgement accuracy on achievement. Teaching and Teacher Education, 3(2), 91-98. https://doi.org/10.1016/0742051X(87)90010-2.

Herppich, H., Praetorius, A.-K., Hetmanek, A., Glogger-Frey, I., Ufer, S., Leutner, D., Behrmann, L., Böhmer, I., Böhmer, M., Förster, N., Kaiser, J., Karing, C., Karst, K., Klug, J., Ohle, A., \& Südkamp, A. (2017). In A. Südkamp \& A. Praetorius (Hrsg.), Diagnostische Kompetenz von Lehrkräften (S. 75-94). Münster: Waxmann.

Herppich, H., Praetorius, A.-K., Hetmanek, A., Glogger-Frey, I., Ufer, S., Leutner, D., Behrmann, L., Böhmer, I., Böhmer, M., Förster, N., Kaiser, J., Karing, C., Karst, K., Klug, J., Ohle, A., \& Südkamp, A. (2018). Teachers' assessment competence: integrating knowledge-, progress-, and product-orientated approaches into a competence-related conceptual model. Teaching and Teacher Education, 76, 181-193. https://doi.org/10.1016/j.tate.2017.12.001. 
Hoffmann, L., \& Böhme, K. (2014). Wie gut können Grundschullehrkräfte die Schwierigkeit von Deutschund Mathematikaufgaben beurteilen? Eine Untersuchung zur Genauigkeit aufgabenbezogener Lehrerurteile auf Klassenebene. Psychologie in Erziehung und Unterricht, 61, 42-55. https://doi.org/10. 2378/peu2014.art05d.

Hößle, C., Hußmann, S., Michaels, J., Niesel, V., \& Nührenbörger, M. (2017). Fachdidaktische Perspektiven auf die Entwicklung von Schlüsselkenntnissen einer förderorientierten Diagnostik. In C. Selter, S. Hußmann, C. Hößle, C. Kniepping, K. Lengnink \& J. Michaelis (Hrsg.), Diagnose und Förderung heterogener Lerngruppen. Theorien, Konzepte und Beispiele aus der MINT-Lehrerbildung (S. 19-37). Münster: Waxmann.

Hoth, J., Döhrmann, M., Kaiser, G., Busse, A., König, J., \& Blömeke, S. (2016). Diagnostic competence of primary school mathematics teachers during classroom situations. ZDM, 48, 41-53. https://doi.org/ 10.1007/s11858-016-0759-y.

Hußmann, S., \& Prediger, S. (Hrsg.). (2014a). Mathe sicher können. 5./6. Schuljahr. Handreichungen für ein Diagnose- und Förderkonzept zur Sicherung mathematischer Basiskompetenzen. Natürliche Zahlen. Berlin: Cornelsen. https://mathe-sicher-koennen.dzlm.de/node/334. Zugegriffen: 28. Dez. 2021.

Hußmann, S., \& Prediger, S. (Hrsg.). (2014b). Mathe sicher können. 5.-7. Schuljahr. Handreichungen für ein Diagnose- und Förderkonzept zur Sicherung mathematischer Basiskompetenzen. Brüche, Prozente und Dezimalzahlen. Berlin: Cornelsen. https://mathe-sicher-koennen.dzlm.de/material/inhalteder-diagnose-und-f\%C3\%B6rderbausteine/online-material-zum-inhaltsbereich-br\%C3\%BCcheprozente. Zugegriffen: 28. Dez. 2021.

Ingenkamp, K. H. (1985). Lehrbuch der pädagogischen Diagnostik. Weinheim: Beltz.

Karst, K., Schoreit, E., \& Lipowsky, F. (2014). Diagnostische Kompetenzen von Mathematiklehrern und ihr Vorhersagewert für die Lernentwicklung von Grundschulkindern. Zeitschrift für pädagogische Psychologie, 28, 237-246. https://doi.org/10.1024/1010-0652/a000133.

Kaufmann, S., \& Wessolowski, S. (2006). Rechenstörungen. Diagnose und Förderbausteine. Seelze: Kallmeyer.

Klauer, K. J. (1973). Revision des Erziehungsbegriffs. Düsseldorf: Pädagogischer Verlag Schwann.

Klauer, K. J. (1978). Diagnostik im Lehr-Lern-Prozess. In K. J. Klauer (Hrsg.), Handbuch der Pädagogischen Diagnostik (Bd. 4, S. 857-871). Düsseldorf: Pädagogischer Verlag Schwann.

Klieme, E., \& Warwas, J. (2011). Konzepte der Individuellen Förderung. Zeitschrift für Pädagogik, 47(11), 805-818.

Kobi, E.E. (1977). Einweisungsdiagnostik - Förderdiagnostik: eine schematisierte Gegenüberstellung. Vierteljahresschrift für Heilpädagogik und ihre Nachbargebiete, 46(2), 115-123.

Kultusministerkonferenz (2014). Standards für die Lehrerbildung: Bildungswissenschaften. Beschluss der Kultusministerkonferenz vom 16.12.2004 i.d. F. vom 12.06.2014. https://www.kmk.org/fileadmin/ Dateien/veroeffentlichungen_beschluesse/2004/2004_12_16-Standards-Lehrerbildung-

Bildungswissenschaften.pdf. Zugegriffen: 28. Dez. 2021.

Kunter, M., Klusmann, U., Baumert, J., Richter, D., Voss, T., \& Hachfeld, A. (2013). Professional competence of teachers: effects on instructional quality and student development. Journal of Educational Psychology, 105(3), 805-820. https://doi.org/10.1037/a0032583.

Leuders, T. \& Loibl, K. (Eds) (2021). Beyond subject specificity-student and teacher thinking as sources of specificity in teacher diagnostic judgments [Special Issue]. RISTAL, 4, 60-70. https://doi.org/10.23770.

Loibl, K., Leuders, T., \& Dörfler, T. (2020). A framework for explaining teachers' diagnostic judgements by cognitive modeling (DiaCoM). Teaching and Teacher Education. https://doi.org/10.1016/j.tate. 2020.103059 .

Luder, R., \& Kunz, A. (2014). Gemeinsame Förderplanung. In R. Luder, A. Kunz \& C. Müller Bösch (Hrsg.), Inklusive Pädagogik und Didaktik (S. 55-71). Zürich: PHZH.

Morris, A. K., Hiebert, J., \& Spitzer, S. M. (2009). Mathematical knowledge for teaching in planning and evaluating instruction: what can preservice teachers learn? Journal for Research in Mathematics Education, 40(5), 491-529. http://www.jstor.org/stable/40539354. Gesehen 28. Dezember 2021.

Moser Opitz, E., \& Nührenbörger, M. (2015). Diagnostik und Leistungsbeurteilung. In R. Bruder, L. Hefendehl-Hebeker, B. Schmidt-Thieme \& H.-G. Weigand (Hrsg.), Handbuch der Mathematikdidaktik (S. 491-512). Berlin/Heidelberg: Springer.

Moser Opitz, E., Pool Maag, S., \& Labhart, D. (2019). Förderpläne: Instrument zur Förderung oder ,,bürokratisches Mittel“? Eine empirische Untersuchung zum Einsatz von Förderplänen. Empirische Sonderpädagogik, 3, 210-224. 
Müller, X., Venetz, M., \& Keiser, C. (2017). Nutzen von individuellen Förderplänen: Theoretischer Fachdiskurs und Wahrnehmung von Fachpersonen in der Schule. Vierteljahresschrift für Heilpädagogik und ihre Nachbargebiete, 86, 116-126. https://doi.org/10.2378/vhn2017.art11d.

Paccaud, A., \& Luder, R. (2017). Participation versus individual support: Individual goals and curricular access in inclusive special needs education. Journal of Cognitive Education and Psychology, 16(2), 205-224. https://doi.org/10.1891/1945-8959.16.2.205.

Peter-Koop, A. (2015). Förderungsdiagnostik mit dem ElementarMathematischen BasisInterview (EMBI) im inklusiven Anfangsunterricht. In A. Peter-Koop, T. Rottmann \& M. Lüken (Hrsg.), Inklusiver Mathematikunterricht in der Grundschule (S. 156-167). Offenburg: Mildenberger.

Philipp, K. (2018). Diagnostic competences of mathematics teachers with a view to processes and knowledge resources. In T. Leuders, K. Philipp \& J. Leuders (Hrsg.), Diagnostic competence of mathematics teachers. Mathematics teacher education (Bd. 11, S. 109-122). Cham: Springer. https://doi.org/ 10.1007/978-3-319-66327-2_6.

Popp, K., Melzer, C., \& Methner, A. (2017). Förderpläne entwickeln und umsetzen (3. Aufl.). München: Reinhardt.

Prediger, S. (2020). Content-specific theory elements for explaining and enhancing teachers' professional growth in collaborative groups. In H. Borko \& D. Potari (Hrsg.), ICMI Study 25 Conference Proceedings. Teachers of mathematics working and learning in collaborative groups (S. 2-14). Lisbon: ICMI.

Prediger, S., Selter, C., Nührenbörger, M., \& Hußmann, S. (2017). Mathe sicher können.5.-8. Schuljahr. Handreichungen für ein Diagnose- und Förderkonzept zur Sicherung mathematischer Basiskompetenzen. Berlin: Cornelsen. https://mathe-sicher-koennen.dzlm.de/material-sek/sachrechnen. Gesehen 28. Dezember 2021.

Ricken, G., \& Schuck, K.D. (2011). Pädagogische Diagnostik und Lernen. In A. Kaiser, D. Schmetz, P. Wachtel \& B. Werner (Hrsg.), Didaktik und Unterricht (S. 110-119). Stuttgart: Kohlhammer.

Rieu, A., Leuders, T., \& Loibl, K.K. (2022). Teachers' diagnostic judgments on tasks as information processing - the role of pedagogical content knowledge for task diagnosis. Teaching and Teacher Education. https://doi.org/10.1016/j.tate.2021.103621.

Rieu, A., Loibl, K., Leuders, T., \& Herppich, S. (2020). Diagnostisches Urteilen als informationsverarbeitender Prozess - Wie nutzen Lehrkräfte ihr Wissen bei der Identifizierung und Gewichtung von Anforderungen in Aufgaben? Unterrichtswissenschaft, 48, 503-529. https://doi.org/10.1007/s42010020-00071-x.

Scherer, P. (2018a). Zwanzigerraum (8. Aufl.). Produktives Lernen für Kinder mit Lernschwächen: Fördern durch Fordern, Bd. 1. Horneburg: Persen.

Scherer, P. (2018b). Addition und Subtraktion im Hunderterraum (8. Aufl.). Produktives Lernen für Kinder mit Lernschwächen: Fördern durch Fordern, Bd. 2. Horneburg: Persen.

Scherer, P. (2019). Multiplikation und Division im Hunderterraum (8. Aufl.). Produktives Lernen für Kinder mit Lernschwächen: Fördern durch Fordern, Bd. 3. Horneburg: Persen.

Scherer, P., \& Opitz, M.E. (2010). Fördern im Mathematikunterricht der Primarstufe. Heidelberg: Spektrum Akademischer Verlag.

Schlee, J. (1985a). Kann Diagnostik beim Fördern helfen? Anmerkungen zu den Ansprüchen der Förderdiagnostik. Zeitschrift für Heilpädagogik, 36(3), 153-165.

Schlee, J. (1985b). Zum Dilemma der heilpädagogischen Diagnostik. Vierteljahresschrift für Heilpädagogik und ihre Nachbargebiete, 54(3), 256-279.

Schlee, J. (2002). Was kann Diagnostik für die pädagogische Praxis leisten? Zu den Ansprüchen der sogenannten Förderdiagnostik. In W. Mutzeck (Hrsg.), Förderdiagnostik. Konzepte und Methoden (S. 181-192). Weinheim: Beltz.

Schlee, J. (2004). Lösungsversuche als Problem. Zur Vergeblichkeit der sogenannten Förderdiagnostik. In W. Mutzeck \& P. Jogschies (Hrsg.), Neue Entwicklungen in der Förderdiagnostik. Grundlagen und praktische Umsetzung (S. 23-38). Weinheim: Beltz.

Schlee, J. (2007). Fördern als planvolle Veränderung Subjektiver Theorien. In W. Mutzeck (Hrsg.), Förderplanung: Grundlagen, Methoden, Alternativen (3. Aufl. S. 178-198). Weinheim: Beltz.

Schlee, J. (2008). 30 Jahre „Förderdiagnostik“ - eine kritische Bilanz. Zeitschrift für Heilpädagogik, 4, $122-133$.

Schrader, F.-W. (1989). Diagnostische Kompetenzen von Lehrern und ihre Bedeutung für die Gestaltung und Effektivität des Unterrichts. Frankfurt: Peter Lang.

Schrader, F.-W. (2013). Diagnostische Kompetenz von Lehrpersonen. Beiträge zur Lehrerbildung, 31(2), $154-165$. 
Shulman, L.S. (1986). Those who understand: knowledge growth in teaching. Educational Researcher, 15(2), 4-14. https://doi.org/10.3102/0013189X015002004.

Spinath, B. (2005). Akkuratheit der Einschätzung von Schülermerkmalen durch Lehrer und das Konstrukt der diagnostischen Kompetenz. Zeitschrift für Pädagogische Psychologie, 19(1/2), 85-95. https://doi. org/10.1024/1010-0652.19.1.85.

Sturm, T., Streit, C., \& Schiefele, C. (2019). Pädagogische Diagnostik und Differenzierung in der Grundschule: Mathe und Deutsch inklusiv unterrichten. Stuttgart: utb.

Van Ophuysen, S. (2010). Professionelle pädagogisch-diagnostische Kompetenz - eine theoretische und empirische Annährung. In N. Berkemeyer, W. Bos, H. G. Holtappels, N. McElvany \& R. SchulzZander (Hrsg.), Daten, Beispiele, Perspektiven. Jahrbuch der Schulentwicklung, (Bd. 16, S. 203-234). Weinheim: Juventus.

Von Aufschnaiter, C., Cappell, J., Dübbelde, G., Ennemoser, M., Mayer, J., Stiensmeier-Pelster, J., Strässer, R., \& Wolgast, A. (2015). Diagnostische Kompetenz - Theoretische Überlegungen zu einem zentralen Konstrukt der Lehrerbildung. Zeitschrift für Pädagogik, 61(5), 738-758.

Wember, F. B. (1998). Zweimal Dialektik: Diagnose und Intervention, Wissen und Intuition. Sonderpädagogik, 28, 106-120.

Wember, F. B. (2018). Mathematik unterrichten - eine subsidiäre Aktivität? Nicht nur bei Kindern mit Lernschwierigkeiten! In P. Scherer (Hrsg.), Zwanzigerraum 8. Aufl. Produktives Lernen für Kinder mit Lernschwächen: Fördern durch Fordern, (Bd. 1, S. 230-247). Horneburg: Persen. 